\title{
Vorwort der Herausgeber
}

Der vorliegende Band verdankt Gestalt und Gehalt besonderen Umständen, die kurz erläutert seien. Die Frage, ob man in den Quellen des römischen Rechts einer kohärent argumentierenden, um begriffliche Klarheit bemühten Rechtswissenschaft begegnet, wurde in der letzten großen, allgemein anerkannten Deutung des römischen Rechts, der Interpolationistik, mit Entschiedenheit verneint. Die Größe der römischen Juristen wurde in ihrer ihrem Judiz folgenden Entscheidungskunst gesehen, d.h. in ihrer Fähigkeit, intuitiv das Richtige zu erkennen. Begriffe, die auf das Gegenteil deuteten, wurden methodisch für interpoliert erklärt.

Die Forschungen von Okko Behrends, die in dem diesem Band angefügten Schriftenverzeichnis umfassend nachgewiesen sind, verfolgen dagegen seit langem das Ziel, die vor der Interpolationistik unumstritten herrschende Meinung, die im römischen Recht ein unübertroffenes Vorbild an Wissenschaftlichkeit verehrte, zu rehabilitieren und sie zugleich, was bisher fehlte, auf historische Gründe zurückzuführen, nämlich einerseits auf die Dauerwirkung der Rom seit seinen Anfängen prägenden, auf die Gewährleistung des Rechtsfriedens gerichteten Auguralreligion, andererseits auf die Rezeption der Rechtslehren zweier hellenistischer Philosophien, erst der Stoa, dann der skeptischen, durch die Pflege der Rhetorik profilierten Akademie des Philon von Larissa.

Sinn und Zweck der Tagung, der ihrer Konzeption von ihrer Initiatorin Cosima Möller eingegeben worden war und der jetzt auf den nachfolgenden Seiten in seinen Ergebnissen ablesbar ist, antworteten auf diese Lage. Aus Anlass des achtzigsten Geburtstags von Okko Behrends sollte - nicht an dem Tag selber, dem 27. Februar 2019, sondern, wie es das römische Recht ermöglicht, an den gleichen Geist aufnehmenden dies utiles, dem 31. Mai und 1. Juni 2019 - , in den gastlichen Räumen der Göttinger Akademie der Wissenschaften in möglichst repräsentativer Weise erkundet werden, wie die Arbeitsergebnisse des Jubilars gegenwärtig beurteilt werden, und zwar in Form eines revisiting seiner Arbeiten durch einen weit gespannten, internationalen und generationenübergreifenden Kreis von Forscherpersönlichkeiten der römischen Rechts- und der allgemeinen antiken Geschichte. Zugleich sollte dem Jubilar Gelegenheit zu einer Stellungnahme gegeben werden, wohl wissend, dass er seine Arbeitsergebnisse nicht als etwas für ihn Abgeschlossenes und hinter sich Gelassenes betrachtet, zu dem er nur noch einmal in Form kurzer Besuche zurückkehren würde, sondern als Forschungsthemen, die ihn, da für ihn immer weiter fortschreitender Klärung fähig und bedürftig, nie losgelassen haben und noch immer erfüllen.

Erreicht wurde dieses Ziel der Tagung mit Hilfe von acht zur Diskussion gestellten Referaten und vorbereiteten Korreferaten zu aussagekräftigen Themen der vier Prägezeiten des römischen Rechts. Der auf die in die Gründungsphase zurückweisende, im stolzen, auf eine Rechtsstellung verweisenden civis Romanus sum! normativ gebliebenen Geschichte des Bürgerrechts folgte eine Würdigung Ciceros unter dem Gesichtspunkt seiner Rolle als des mit Abstand wichtigsten Zeugen, der für die 
Rezeption der beiden für das römische Recht grundlegend gewordenen Philosophien angeführt werden kann. Für die Kaiserzeit schloss sich zunächst die Analyse des Phänomens der auf Schulkontroversen reagierenden Mittelmeinungen sinnvoll an, da deren Auftreten darauf deutet, dass sich in den mit dem Prinzipat des Augustus erscheinenden beiden Rechtsschulen die beiden philosophischen Richtungen in einer nach Ausgleich rufenden Weise fortgesetzt haben. Auch das letzte Thema wahrte den Zusammenhang: Es galt Justinian und seiner ihn zum „Juristenkaiser“ machenden Fähigkeit, die in den Quellen stehengebliebenen Schulkontroversen für seine auf Widerspruchsfreiheit gerichtete Kodifikation sachkundig zu entscheiden.

Am Ende ergab sich in der Tat ein Gesamtbild, das angesichts der Zahl und des Ranges der Teilnehmer als eine Art repräsentativer Momentaufnahme des gegenwärtigen Meinungsstandes betrachtet werden kann.

Hinsichtlich der Wissenschaftlichkeit des römischen Rechts herrschte schließlich weitgehend Konsens. Dass die Gründungsphase als Berechtigungssystem des freien Bürgers das Vindikationsmodell überliefert, dass Cicero für die Rechtsgeschichte ein höchst ernst zu nehmender Zeuge ist, dass die beiden Schulen in ihren Lehren grundverschiedene Sichtweisen vertreten und dass Justinian deren letzte Differenzen zugunsten einer systematisch gedachten Rechtsordnung beseitigen wollte, wurde von allen in seiner ganzen Bedeutung anerkannt. Auch hinsichtlich der beiden hellenistischen Philosophien, die das bisher in jeder Deutung als Einheit empfundene römische Recht für die Rekonstruktion seiner inneren Geschichte in zwei grundverschiedene Systeme aufzuteilen gebietet, hatten die Beiträge der drei letzten Abteilungen unter den Symposiasten viel Aufgeschlossenheit erzeugt, die sich zum Teil in Zustimmung äußerte, zum Teil weitere Klärungen forderte. In zwei Fällen war der Klärungsbedarf so grundsätzlich, dass sich der Jubilar nicht wie sonst auf eine Stellungnahme beschränkte, sondern zu beiden selbstständige Studien vorlegte. Die erste gilt dem Ursprung des Identität verleihenden Qualitativen in einer Mittelmeinung: Ist es ein Beitrag der Stoa, deren Providenz die Gegenstände der Dingwelt von innen mit Sinn erfüllt, oder einer der Skepsis, die grundsätzlich deren sich dem Menschen zeigende Außenseite entscheiden lässt? Die zweite ging auf die Frage ein, in welcher Weise die Skepsis die Stoa in der Rechtslehre abgelöst hat: War es ein destruktives Verwerfen der Vorgängerin, das alle Beziehungen abbrach, oder ein achtungsvolles, durch sichtbar gehaltene Unterschiede literarische Kontinuität wahrendes Herausarbeiten des Neuen?

Die beiden Tage waren durch schönstes Frühlingswetter begünstigt, was dem geselligen Begleitprogramm in besonderer Weise zugutekam. Am Abend des ersten Tages hatte der Jubilar mit seiner Ehefrau Helge Behrends die Teilnehmer in sein Haus gebeten, das von seinem oberen Garten einen Blick über das weite Leinetal gewährt. Der Abschlussabend führte dann im Rahmen einer Exkursion an die Weser zu einem - mit der Kopie einer Speisekarte von 1874 ein wenig Jhering nachahmenden - „Diner en l'honneur de l'Esprit du Droit Romain“ im Rokokosaal des am gerade neu gefluteten Hafenbecken der Hugenottenstadt Karlshafen gelegenen, der Göttinger Universität vielfach verbundenen Hotel Schwan. Es war denn auch in der Tat - die an 
jenem Abend gesprochenen Worte bezeugten es - die von allen geteilte Verehrung des großen, noch vielverheißenden Forschungsgegenstandes Römisches Recht, dessen sich jetzt in so vielen Kontroversen abzeichnende „dialogische“ Offenheit ihm ersichtlich keinen Eintrag getan hat, die dem Symposion eine über alle alten und neuen Meinungsverschiedenheiten hinweg fruchtbare und diskussionsbereite Atmosphäre verliehen hat. Von ihr getragen hat der Jubilar denn auch an jenem Abend alle in zehn Jahren an gleichem Ort erneut zu einem entsprechenden Diner eingeladen, natürlich sub conditione generali Jacobaea, was uns ein kurzes Gedenken erlaubt.

Rolf Knütel (23.12.1939 - 25.9.2019), der als jemand, der am Ende des gleichen Jahres seinen achtzigsten Geburtstag gefeiert hätte, die Sitzungsleitung des ersten Themas des Symposions übernommen hatte, musste seine Teilnahme absagen. Durch sein Werk, nicht zuletzt durch die von ihm geprägte, viele Romanisten zur Mitarbeit heranziehende, neue Übersetzung des Corpus Iuris Civilis, und sein den allermeisten Teilnehmern bekanntes Wesen hatte er auch als Abwesender seinen Anteil daran, dass sich jene den „Geist des römischen Rechts“ verehrende Atmosphäre in zukunftsfähiger Weise bilden konnte.

Abschließend gilt es Dank zu sagen: Die Göttinger Akademie der Wissenschaften hat das dem wissenschaftlichen Werk ihres langjährigen Mitglieds geltende Symposion und die Drucklegung seiner Ergebnisse entscheidend gefördert. Ihr, ihrem Mitarbeiterstab und insofern an erster Stelle ihrer damaligen Generalsekretärin Dr. Angelika Schade haben wir daher in erster Linie zu danken. Für weitere Förderung gilt unser Dank der Göttinger Juristenfakultät, dem Göttinger Institut für Grundlagen des Rechts sowie der Strohmeyer-Stiftung, die im Rahmen ihres Satzungszweckes der Förderung des rechtshistorischen Nachwuchses einen Beitrag geleistet hat.

Ein besonderer persönlicher Dank gilt unserer Kollegin Eva Schumann, die uns vielfältige Unterstützung hat zuteilwerden lassen und ihrer Sekretärin, Frau Petra Wienands, für die umsichtige Vor- und Nachbereitung des Symposions. Für die Vorbereitung der Druckfassung des Bandes gebührt Dank und Anerkennung Frau Hendrike Diesselhorst, wissenschaftliche Mitarbeiterin an der Freien Universität Berlin, und Herrn Caspar Manntz, wissenschaftlicher Mitarbeiter ebendort.

Dem aufmerksamen Leser wird nicht entgangen sein, dass das Vorwort nicht nur mit dem Jubilar abgestimmt ist, sondern wir ihm auch zu einem guten Teil das Wort überlassen haben. Wer könnte in die Forschungsergebnisse, die nach ihren Grundannahmen und Einzelthesen Gegenstand der kritischen Überprüfung durch die auf den folgenden Seiten dokumentierten Beiträge sind, besser einführen als ihr an ihnen, wie seine Entgegnungen und die beiden jeweils um vertiefte Klärung aufgeworfener Fragen bemühten Abhandlungen zeigen, intensiv fortarbeitender Urheber?

Berlin, Köln und Kiel, im Juni 2020

Cosima Möller

Martin Avenarius

Rudolf Meyer-Pritzl 
